\title{
Direct observation and evolution of electronic coupling between organic semiconductors
}

\author{
Sameer Vajjala Kesava $\odot^{*}$ and Moritz K. Riede $\oplus^{\dagger}$ \\ Department of Physics, University of Oxford, OX1 3PU, England, United Kingdom
}

(Received 12 August 2020; revised 17 November 2020; accepted 24 November 2020; published 6 January 2021)

\begin{abstract}
The electronic wave functions of an atom or molecule are affected by its interactions with its environment. These interactions dictate electronic and optical processes at interfaces, and is especially relevant in the case of thin film optoelectronic devices such as organic solar cells. In these devices, charge transport and interfaces between multiple layers occur along the thickness or vertical direction, and thus such electronic interactions between different molecules_-same or different—are crucial in determining the device properties. Here, we introduce an in situ spectroscopic ellipsometry data analysis method called differential analysis in real time (DART) with the ability to directly probe electronic coupling due to intermolecular interactions along the thickness direction using vacuum-deposited organic semiconductor thin films as a model system. The analysis, which does not require any model fitting, reveals direct observations of electronic coupling between frontier orbitals under optical excitations leading to delocalization of the corresponding electronic wave functions with thickness or, equivalently, number of molecules away from the interface in C60 and MeO-TPD deposited on an insulating substrate $\left(\mathrm{SiO}_{2}\right)$. Applying the same methodology for $\mathrm{C} 60$ deposited on phthalocyanine thin films, the analyses shows strong, anomalous features-in comparison to $\mathrm{C} 60$ deposited on $\mathrm{SiO}_{2}$-of the electronic wave functions corresponding to specific excitation energies in C60 and phthalocyanines. Translation of such interactions in terms of dielectric constants reveals plasmonic type resonance absorptions resulting from oscillations of the excited state wave functions between the two materials across the interface. Finally, reproducibility, angstrom-level sensitivity, and simplicity of the method are highlighted showcasing its applicability for studying electronic coupling between any vapor-deposited material systems where real-time measurements during thin film growth are possible.
\end{abstract}

DOI: 10.1103/PhysRevMaterials.5.015601

\section{INTRODUCTION}

The electronic wave functions of an atom in a solid depend on the positions of the electrons and the nuclei. Changes in the energy levels occur due to the overlap of the ground state electronic wave functions primarily of the frontier orbitals between neighboring atoms or molecules, and determines the optoelectronic properties of a solid, e.g., leading to the emergence of bands in semiconductors [1,2]. Understanding how the energy levels in an atom evolve from isolation to a many-atom solid is one of the most important fundamentals of solid state physics required for explaining the properties of solids such as metals, semiconductors, and insulators.

In the case of organic semiconductors, comprised typically of large molecules, understanding the effects of intermolecular interactions on the electronic wave functions is crucial for understanding the physics at interfaces since the interfacial phenomena, e.g., transfer of a charge between a donor and an acceptor in solar cells, are affected by the local environment [3-8] to which the frontier orbitals react. This is relevant not just for organic semiconductors but any electronic material such as conductive oxides, chalcogenides, and

\footnotetext{
*Corresponding author: sameer.vajjalakesava@physics.ox.ac.uk

${ }^{\dagger}$ moritz.riede@physics.ox.ac.uk
}

metals forming the layers of optoelectronic thin film devices where interfacial processes play a critical role in the device properties, and especially more in the case of vertical devices (where charge transport occurs along the thickness direction in contrast to thin film field-effect transistors). In situ optical spectroscopic techniques such as in situ differential reflectance spectroscopy (DRS) $[9,10]$ and reflection anisotropy spectroscopy (RAS) [11-13] have been around for many years and offer a nondestructive way of probing such intermolecular interactions. in situ DRS has been used to study electronic coupling and its evolution with thickness, for example, between flat-lying tin(II)-phthalocyanine ( $\mathrm{SnPc}$ ) molecules [10] and between quaterrylene molecules [9] deposited under vacuum. These works demonstrate the strength of in situ optical spectroscopic techniques, but also one of the current limitations of DRS and RAS. While it is possible to infer electronic coupling in the perpendicular direction, for example, between quaterrylene molecules and the underlying gold layer (as a metal substrate) [9], due to the near-normal incidence of the probe light in DRS (and in RAS), the data obtained is primarily that of the in-plane component of the dielectric constants [9], since the orientation of the electric field of the probe light is nearly all in the plane of the film. Being able to directly access the out-of-plane interactions of molecules would complement DRS/RAS and open up new avenues in probing and characterizing electronic coupling in interacting systems essential for further 
expanding the understanding of the physics of thin film optoelectronic devices. For example, in many organic electronic devices, key processes happen at planar interfaces parallel to the substrate, e.g., this is where the generation of free charge carriers happens in planar heterojunction organic solar cells.

One experimental technique which can probe this out-ofplane direction is in situ spectroscopic ellipsometry (iSE), also sometimes referred to as "real-time" SE. This is possible firstly because of the high angle of incidence employed during measurements (typically $65^{\circ}$ ). Secondly because of the use of polarized light, where the amplitude and phase change of the electric field upon interaction with the thin film are measured $[14,15]$. The combination of these parameters carry more information than just the intensity measured in typical optical spectroscopic techniques, and which is also the most significant drawback of $\mathrm{x}$-ray techniques where phase information is lost. Thus, SE is a thin film characterization technique typically used to characterize the primary optical excitations in terms of optical properties: dielectric constants or refractive indices that represent the quality of the thin films $[14,15]$. In the case of organic solar cells, the technique is typically used to characterize the active layers for understanding structure-property relationships [16-19], and for optical simulations to model absorption profiles within a device [20].

iSE is an advanced application of SE which makes it possible to study the growth and evolution of the optical properties of multilayered thin films, e.g., semiconductors deposited by thermal evaporation under vacuum or other vapor-deposition methods [21-23]. (i)SE, in general, has an angstrom-level sensitivity towards changes in thicknesses. The standard SE analysis procedure, or SSE in short, for obtaining optical properties from the iSE data relies on fitting a dielectric function model (using, for example, Gaussian and Lorentzian functions) to the data, and the typical analysis is initiated with the assumption that the film has the same properties along its thickness [14,15]. However, robust and confident in-depth analysis such as variation of dielectric constants along the thickness direction is not possible because of lack of additional data (only one angle of incidence in iSE against different angles of incidence as is possible in ex situ SE including at normal incidence). Thus, the information obtained from SSE analysis is determined not only by the dielectric functions used during the fitting but also the type of model used to analyze single or multilayered thin films. Moreover, from model fitting, even if any anomalies are seen in the dielectric constants especially along the growth direction due to interactions with other materials at the interfaces, the question of whether these anomalies are real or artifacts from fitting is posed. To ascertain the analysis, additional optical information such as about the in-plane dielectric constants obtained from in situ DRS $[9,10]$ is essential.

In this work, we present an experimental methodology using iSE coupled with a data analysis method for studying the evolution of the electronic wave functions pertaining to the optical excitations along the thickness or vertical direction as a function of thickness or, equivalently, number of molecules in vacuum-deposited thin films. By an empirical comparison to the optical properties $(n+j k)$, we show that the iSE data analysis method extracts information representative of the same for every layer (thickness resolution down to $1 \AA$ possible) deposited. The analysis does not involve any model fitting and is only based on tracking the differential change in the iSE data. Any changes in the electronic wave functions corresponding to the frontier orbitals under excitations due to overlap with that of the neighboring molecules-both same and different species-in the growth (thickness) direction is reflected in the information obtained from the analysis. We demonstrate the ability of the method by its application to the study of vacuum-deposited organic semiconductor thin films using fullerene (C60), boron subphthalocyanine chloride (SubPc), boron sub-2,3-naphthalocyanine chloride (SubNc) and N,N,N',N'-Tetrakis-(4-methoxyphenyl)benzidine (MeOTPD) on different underlying layers.

We also evaluate and discuss the implications of these observations by complementing the analysis with a systematic approach for obtaining dielectric constants along the thickness direction using SSE data analysis, i.e., model fitting. Moreover, we demonstrate the angstrom-level sensitivity, simplicity, and reproducibility of the method. Significant modulations in the optical response due to electronic coupling have been observed and the method reported. Finally, we conclude with the general applicability of this method for studying other systems where electronic coupling through intermolecular interactions are crucial.

\section{EXPERIMENTAL SECTION}

The organic semiconductors SubPc (> 99\%, Lumtec), SubNc ( $>$ 99\%, Lumtec), C60 ( 99.999\%, CreaPhys), molybdenum oxide (MoOx) (> 99.998\%, Lumtec) and MeOTPD ( $>99 \%$, Lumtec) were used as purchased. Substrates silicon wafer (with $23 \mathrm{~nm} \mathrm{SiO}_{2}$ on top, purchased from J.A.Woollam, for calibrating the ellipsometer) and ultrasmooth quartz substrate (WZWOPTICAG) were cleaned with soap and deionized water, and then sonicated in acetone and isopropanol (in this order) at $50{ }^{\circ} \mathrm{C}$ for $20 \mathrm{~min}$ before drying with an air gun. Then the substrates were UV-ozone treated for $10 \mathrm{~min}$ and loaded into the vacuum chamber (CreaPhys) via a nitrogen glovebox. A Woollam RC2 spectroscopic ellipsometer was mounted at $\sim 65^{\circ}$ angle of incidence onto the vacuum chamber. The thin films were deposited on the loaded substrates by thermal evaporation of the organic semiconductors (at rates $0.1-0.5 \AA / s$ ). The in situ measurements (210-1690 nm or $0.7-5.9 \mathrm{eV})$ were carried out with an acquisition time which ranged from 4-10 s or measurement of data for every $\sim 1-2 \AA$ increase in thickness.

SSE for deriving the refractive indices of each layer through model fitting to the data (and described in Sec. S2 in the Supplemental Material [24]) was carried out in CompleteEASE software from J.A.Woollam company. ex situ SE data of some of the thin films after deposition was obtained, where required, at $55^{\circ}, 65^{\circ}$, and $75^{\circ}$ angles of incidence. The $\psi$ and $\Delta$ time series values measured through CompleteEASE were exported into text format and analyzed in python using the equation described in the Results and Discussion section. 


\section{RESULTS AND DISCUSSION}

\section{A. DART}

In standard SE measurements of thin films, the data measured are the electric field amplitude ratio $\psi$ and phase difference $\Delta$ as a function of wavelength $(\lambda)$ or energy $(E)$ of the probe light defined as the ellipsometric ratio $\rho[14,15]$

$$
\rho=\tan \psi(E) e^{-j \Delta(E)} .
$$

During iSE, $\rho$ is measured as a function of time $t$ or, equivalently, thickness $d$ in the case of monitoring the growth of vacuum-deposited thin films, which in our case are organic semiconductors. A first derivative of Eq. (1) with respect to time (or $d$ ) yields the following equation:

$$
\frac{\delta \rho}{\delta t}=e^{-j \Delta}\left[(\sec \psi)^{2} \times \frac{\delta \psi}{\delta t}-j \times \tan \psi \times \frac{\delta \Delta}{\delta t}\right] .
$$

When the time interval between measurements is constant, which is typically the case in in situ measurements, Eq. (2) simplifies to

$$
\delta \rho_{t}=e^{-j \Delta_{t}}\left[\left(\sec \psi_{t}\right)^{2} \times \delta \psi_{t}-j \times \tan \psi_{t} \times \delta \Delta_{t}\right],
$$

where

$$
\operatorname{real}(\delta \rho)=\cos \Delta_{t}\left(\sec \psi_{t}\right)^{2} \delta \psi_{t}-\sin \Delta_{t} \tan \psi_{t} \delta \Delta_{t},
$$

$\operatorname{imaginary}(\delta \rho)=-\left[\sin \Delta_{t}\left(\sec \psi_{t}\right)^{2} \delta \psi_{t}+\cos \Delta_{t} \tan \psi_{t} \delta \Delta_{t}\right]$.

The measured iSE data, $\psi$ and $\Delta$, can be numerically differentiated for $\delta \psi$ and $\delta \Delta$ yielding $\delta \rho$ at time $t$ or thickness $d$.

iSE is a highly sensitive technique, sensitive to changes in the thickness direction of a surface $[14,15]$ (also referred to as out-of-plane or $z$ direction). The in-plane $/ x y$ direction, probed in UV-visible absorption measurements, is defined as the plane of the film (see Fig. S1). During iSE, the probe light passes through all the layers for every time point for which $\rho$ is measured. Since the thin film deposition or the direction of growth during iSE is along the $z$ direction of the film, $\delta \rho$ then is the rate of change of the optical response $\rho$ of the thin film, which is primarily a function of the change in the optical properties of the thin film in the $z$ direction, namely out-of-plane refractive indices $\mathrm{N}_{z}\left(n_{z}+j k_{z}\right)$ or, equivalently, dielectric constants $\epsilon_{z}\left(\epsilon_{1 z}+\mathrm{j} \epsilon_{2 z}\right)$. A basic derivation of $\delta \rho$ in terms of $\mathrm{N}_{x y}$ (in-plane refractive indices of the film), $\mathrm{N}_{z}$ and $d$ is given Sec. S1 of the Supplemental Material. However, for a small, differential change in thickness $\delta d, \delta \rho$ can also be interpreted as the optical response of the incoming layer $\delta d$ that will be deposited on the substrate with the thin film at thickness $d$ as exemplified in Figs. 1 and 2. The following results are based on this method called differential analysis in real time (DART).

\section{B. C60 and MeO-TPD on $\mathrm{SiO}_{2}$}

Figure 1(a) shows the real and imaginary parts as well as modulus of $\delta \rho$ of C60 deposited on an optically smooth quartz substrate $\left(\mathrm{SiO}_{2}\right)$; optically smooth implies that for the range of probe wavelengths $(210-1690 \mathrm{~nm})$, the roughness is on a sub-angstrom level. Thickness $d$ of the film is obtained from SSE analysis (see Sec. S2). $\delta \rho$ has been calculated for every $1 \mathrm{~nm}$ interval, i.e., $\delta d=1 \mathrm{~nm}$, for all the data presented in this work. Firstly, to understand the meaning of real and imaginary values of $\delta \rho$, we compare $\delta \rho$ 's value calculated for the first $\delta d$ layer deposited on a blank substrate, i.e., at $d=0 \mathrm{~nm}$, to the $k$ and $n$ values of the C60 film (at $13 \mathrm{~nm}$ thickness) in Fig. 1(b) obtained from SSE analysis using Gaussian functions (commonly referred to as oscillators). Such a function/oscillator can be used to fit to the absorption peaks which represent the sum of the main electronic transition and vibrionic progressions. Examining the $\delta \rho$ profile of the first nanometer deposited, it can be seen that the $\operatorname{real}(\delta \rho)$ and imaginary $(\delta \rho)$ appear to represent $k$ and $n$ values, respectively. The same observation can be seen for MeO-TPD (deposited on optically smooth quartz) in Fig. 2. Thus, $\delta \rho$ represents the optical properties of a layer $\delta d$, and its corresponding real (negative) and imaginary parts will be hereinafter referred to as $\delta \rho_{k}$ and $\delta \rho_{n}$, respectively.

SE probes the optical excitations of a system in terms of extinction coefficient $k$. Examining $\delta \rho_{k}$ in Fig. 1(a) for the first $1 \mathrm{~nm}$ layer, which can be approximated as a monolayer of C60 molecules that is deposited on a blank substrate $(d=$ $0 \mathrm{~nm}$ ), we observe that the strongest responses are centered at 3.6, 4.6, and $5.5 \mathrm{eV}$, which correspond to the allowed, primary transitions $T_{1 u} \leftarrow A_{1 g}$ with the strongest absorption in C60 [25-29]; also reflected in the extinction coefficient plot in Fig. 1(b). This first nanometer data can be assumed as the average optical response of one $\mathrm{C} 60$ molecule deposited onto the $\mathrm{SiO}_{2}$ substrate. As more $\mathrm{C} 60$ deposits, we observe that the optical response is not the same for every additional nanometer being deposited. The magnitude gradually decreases and the peaks redshift. This can be attributed to the intermolecular interactions between $\mathrm{C} 60$ molecules in the form of electronic coupling arising from overlap (along the thickness direction) of the frontier orbitals corresponding to the respective primary transitions in C60.

A consequence of this coupling is the delocalization of the corresponding electronic wave functions under excitation (or excited state wave functions) in C60, - a well-known phenomenon in C60 [30-33], - and analogous to band formation in inorganic semiconductors or J-type interaction [34,35] causing a gradual red-shift (along with polarization effects $[36,37]$ ) of the primary transitions with deposition. If there were no electronic coupling between the molecules, and consequently no delocalization, the response $\delta \rho$ should be the same for every deposition resulting in the same $\delta \rho$ for every $\delta d$. A constant $\delta \rho$ implies $\delta N_{z}=0$ (from Eq. S1.8) which is what is approximately observed for MeO-TPD for energies corresponding to the $\pi-\pi^{*}$ transitions at $3.4 \mathrm{eV}$ in Fig. 2(a). The similar optical response at the $\pi-\pi^{*}$ transition energy for all thicknesses in MeO-TPD implies that the corresponding excited state wave function, unlike in C60, is highly localized indicating weak electronic coupling with neighboring molecules at this energy. This is reflected in the extremely low mobilities of MeO-TPD [38,39]. However, the overlap of wave functions of the frontier orbitals in $\mathrm{MeO}$ TPD occurs at higher energies as seen from the changes in $\delta \rho$ with deposition. These energies are higher than the ionization potential of MeO-TPD ( 5.1 eV [40]), and possibly 

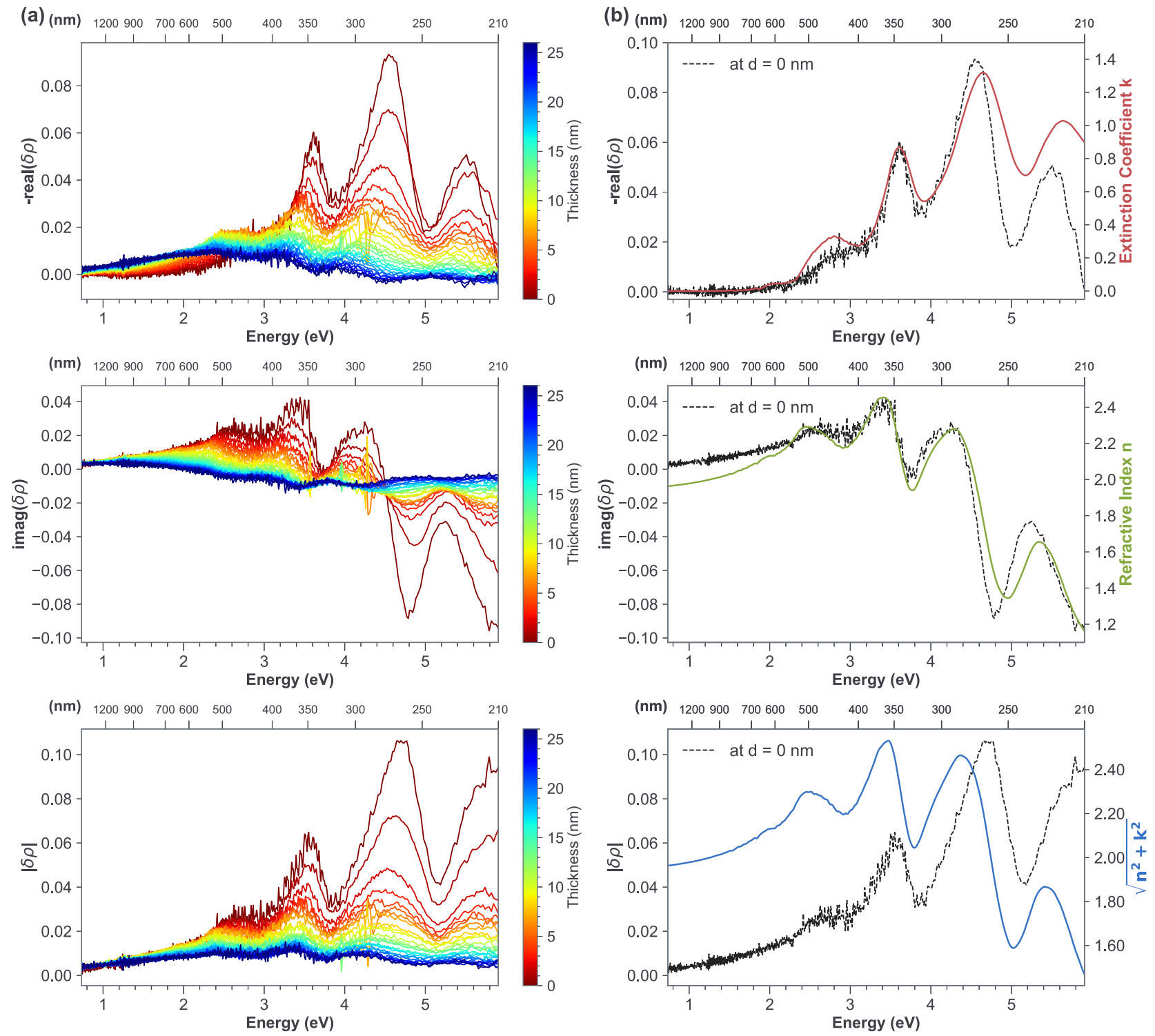

FIG. 1. (a) DART analysis showing the real (negative) and imaginary parts as well as the modulus of $\delta \rho$ of C60 deposited on an optically smooth quartz substrate $\left(\mathrm{SiO}_{2}\right)$, calculated for a differential change of $\delta d=1 \mathrm{~nm}$ in $\mathrm{C} 60$ thickness. (b) Comparison of the isotropic extinction coefficient $k$, refractive index $n$, and the modulus of $n+j k$ of the same C60 film derived from SSE analysis at $d=13 \mathrm{~nm}$ to the negative real, imaginary and modulus of $\delta \rho$, respectively, of the first $1 \mathrm{~nm}$ layer deposited on the blank substrate (at "thickness" $d=0 \mathrm{~nm}$ ), taken from the DART analysis in (a).

correspond to transitions from energy levels below highest occupied molecular orbital (HOMO) to lowest unoccupied molecular orbital (LUMO) and above.

The dynamics of the three electronic transitions of C60 [Fig. 1(a)] seem to be different. Initially, the $4.6 \mathrm{eV}$ transition has the highest strength. With addition of C60 (the film grows smoothly, see Sec. S2) the strength gradually decreases and goes below the $3.6 \mathrm{eV}$ transition as shown in Fig. S2, i.e., the rate of decrease of $\delta \rho$ of the $4.6 \mathrm{eV}$ transition (also $5.5 \mathrm{eV}$ ) is higher compared to the $3.6 \mathrm{eV}$ transition at least for the initial few nanometers; rate of change of $\delta \rho$ can itself be estimated from the second derivative of Eq. (2) but not shown here because of the noisy output of the calculation. Additionally, a kink in the $4.6 \mathrm{eV}$ transition appears at $d=$ $5 \mathrm{~nm}$ and persists until $13 \mathrm{~nm}$ or approximately a layer of
13 C60 molecules thick/high; the origin of this is not clear. It is possible that some sort of hybridization of the frontier orbitals corresponding to the $4.6 \mathrm{eV}$ transition has occurred leading to its manifestation as a kink. Finally, analysis of the rates of redshift of the transition peaks is shown in Fig. 3. The $4.6 \mathrm{eV}$ transition not only appears to decay faster (along with a kink) but also redshifts faster $(26 \mathrm{meV} / \mathrm{nm})$ compared to the $3.6 \mathrm{eV}(18 \mathrm{meV} / \mathrm{nm})$ and the $5.5 \mathrm{eV}(15 \mathrm{meV} / \mathrm{nm})$ transitions. The broad, and weak, feature around $2.4 \mathrm{eV}$ (below $3.0 \mathrm{eV}$ ), also redshifting with the addition of $\mathrm{C} 60$, is visible from the deposition of the first nanometer itself, and corresponds to a forbidden transition that occurs due to mixing of the vibronic states with the forbidden states [25-29]. Such redshifts of the transitions with thickness occurring due to coupling is a common feature in organic semiconductors as shown for 

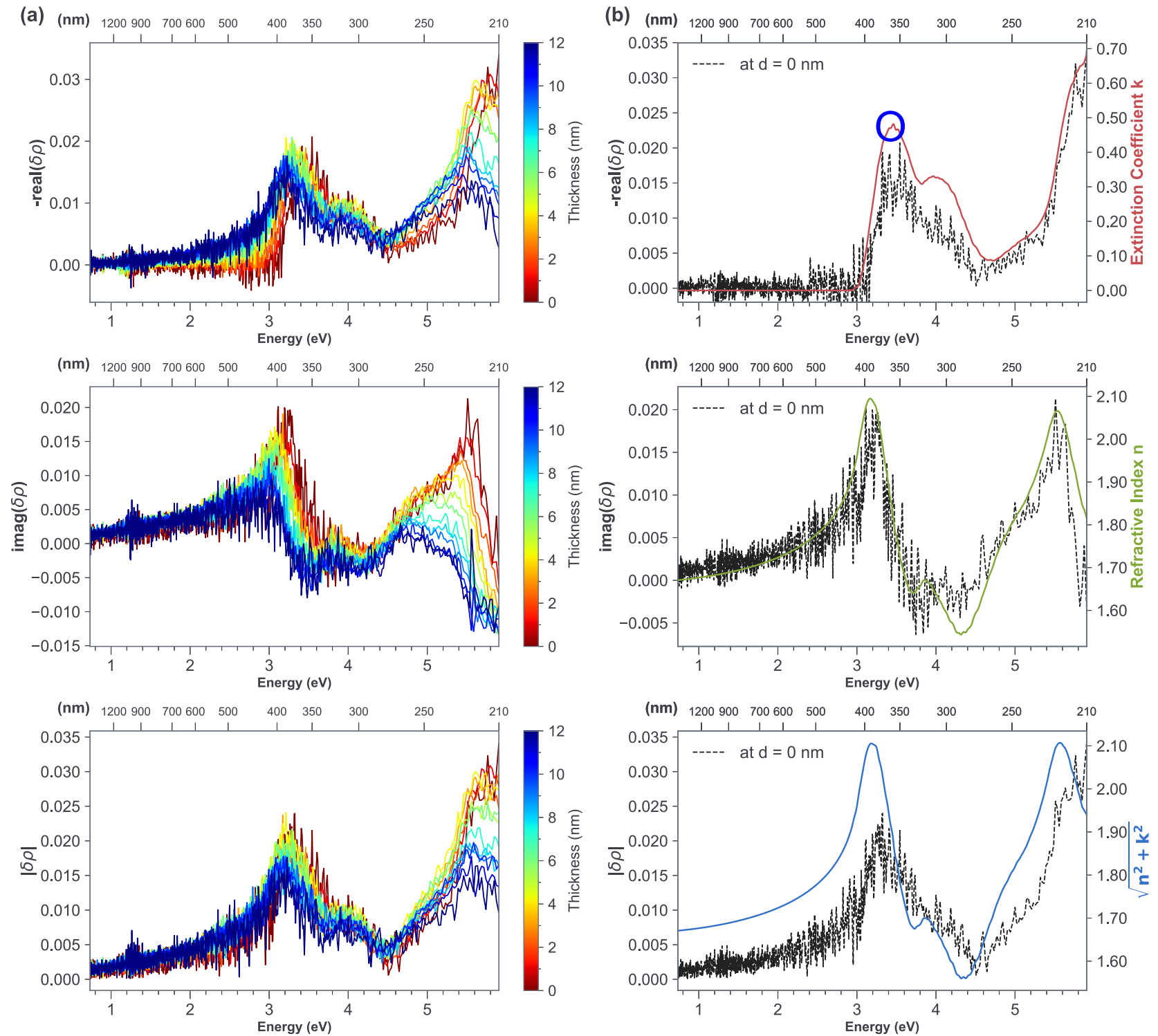

FIG. 2. (a) DART analysis showing the real (negative) and imaginary parts as well as the modulus of $\delta \rho$ of MeO-TPD deposited on an optically smooth quartz substrate $\left(\mathrm{SiO}_{2}\right)$ calculated for a differential change of $\delta d=1 \mathrm{~nm}$. (b) Comparison of the isotropic extinction coefficient $k$, refractive index $n$, and the modulus of $n+j k$ of the same MeO-TPD film derived from SSE analysis at the final thickness of $12 \mathrm{~nm}$ to the $\operatorname{real}(\delta \rho)$, imaginary $(\delta \rho)$, and modulus $(\delta \rho)$ values, respectively, of the first $1 \mathrm{~nm}$ layer deposited on the blank substrate ("thickness" $d=0$ nm), taken from the DART analysis in (a). The $\pi-\pi^{*}$ transition at $3.4 \mathrm{eV}$ is circled blue in the plot of extinction coefficient $\mathrm{k}$ in (b).

other systems such as SnPc [10] and $\alpha$-quaterthiophene [13]. Finally, in the energy range corresponding to the transparent region of $\mathrm{C} 60$ (from $\sim 750 \mathrm{~nm}$ or $1.7 \mathrm{eV}$ ), any such coupling should be absent, and is reflected in the similar and flat profile of $\delta \rho$.

Such variation of $\delta \rho$ with thickness shows that the optoelectronic properties of $\mathrm{C} 60$ or any such interacting systems is not uniform along the $z$ direction. Moreover, because the wave functions of C60 delocalize, the optical response $\delta \rho$ of a layer $\delta d$ deposited at time $t$ cannot be solely assigned to that layer $\delta d$ [30-33]. The C60 molecules at the top and bottom of the film will be experiencing different environments, and thus coupling, compared to the molecule in the middle. Hence, the overall $\delta \rho$ could be the sum of all the changes in the underlying bulk layer (of thickness $d$ ) along with the layer $\delta d$ deposited at time $t$. This emphasizes that much care is needed when determining the optical constants along the thickness direction from SSE analysis thus highlighting its shortcomings; the values obtained are representative mainly of the properties along the $x y$ or in-plane direction. Thus, the DART method can be used to track the optical properties of vacuum-deposited thin films in real time without the need for real-time model fitting of the iSE data, which becomes highly complicated for multlilayered absorbing and anisotropic materials. However, the drawback is that the information obtained will be representative primarily of that of the optical properties in the out-of-plane direction. And to obtain the dielectric constants in interacting systems especially in 


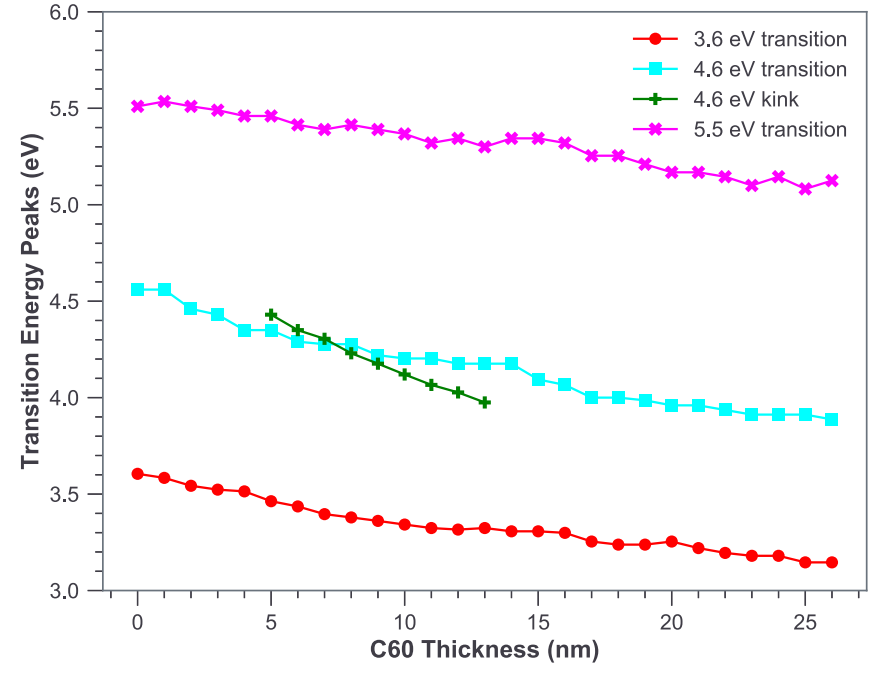

FIG. 3. Energy values of the C60 primary transition peaks as a function of $\mathrm{C} 60$ thickness obtained from the DART results in Figs. 1 and S2.

the out-of-plane direction, as the results show, iSE will be insufficient and will need to used in conjunction with other methods such as in situ DRS [9] to decouple in-plane and out-of-plane optical properties.

\section{SubPc}

DART analysis of a SubPc film deposited on a silicon wafer is shown in Fig. 4(a) (only $|\delta \rho|$ is shown from here on as it represents $n$ and $k$ together). From the evolution of the data with thickness, we see that a strong electronic coupling between subsequent SubPc layers occurs at energies above $3 \mathrm{eV}$, i.e., between frontier orbitals lying much higher than for the $2.1 \mathrm{eV} \pi-\pi^{*}$ transition [41,42]. Figure 4(a).2 is a magnified view of the data in Fig. 4(a).1 showing the evolution of the optical response of the $\pi-\pi^{*}$ transition (the trough in $|\delta \rho|$ ) at $2.1 \mathrm{eV}$ in SubPc. From the DART analyses of MeO-TPD and C60, a constant $\delta \rho$ with thickness implies negligible or weak delocalization. Thus, the electronic coupling between frontier orbitals corresponding to the $\pi-\pi^{*}$ transition in SubPc appears to occur only until the third or the fourth nanometer after which $|\delta \rho|$ at $2.1 \mathrm{eV}$ remains constant indicating that the extent of delocalization of this excited state wave function is small, i.e., up to a maximum of $4 \mathrm{~nm}$ compared to at least $26 \mathrm{~nm}$ in C60 (Fig. 1). Corresponding $\mathrm{SSE}$ analysis of the completed SubPc thin film $(16 \mathrm{~nm})$ for the average in-plane and out-of-plane optical constants is shown in Figs. S3(e) and 3(f). As seen from this figure, an anisotropic model best describes the growth of SubPc (the mean squared error of the fit decreases from 5.4 for an isotropic to 2.1 for an anisotropic model), and a weak transition (O1) at $\sim 2 \mathrm{eV}$ can also be seen.
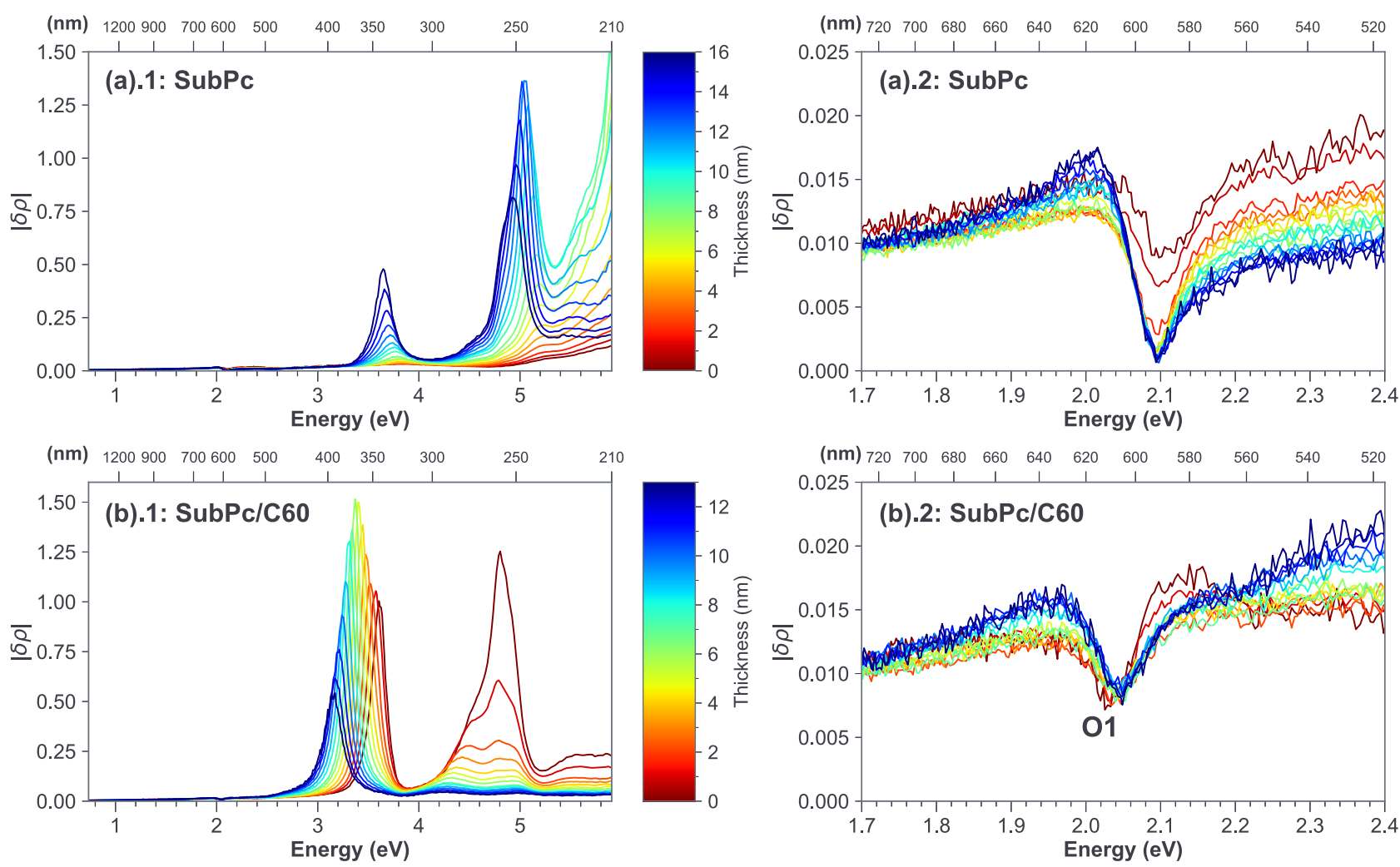

FIG. 4. (a).1 DART analysis of a SubPc film deposited on a $\mathrm{Si} / \mathrm{SiO}_{2}$ substrate. (a).2 Magnified view of the same DART results in (a).1 showing the optical response corresponding to the $\pi-\pi^{*}$ transition $[41,42]$ in SubPc for clear visualization. (b). 1 DART analysis of C60 deposited onto the SubPc film in (a). (b).2 Magnified view of the data of (b). 1 around $2.1 \mathrm{eV}$ for clear visualization. The optical response corresponding to the excitations into SubPc excitonic states is indicated by the label O1, which corresponds to the transition in Fig. S3(f). All calculations were carried out for a differential change of $\delta d=1 \mathrm{~nm}$. 
(a): $\varepsilon_{z}-\mathbf{C 6 0}$

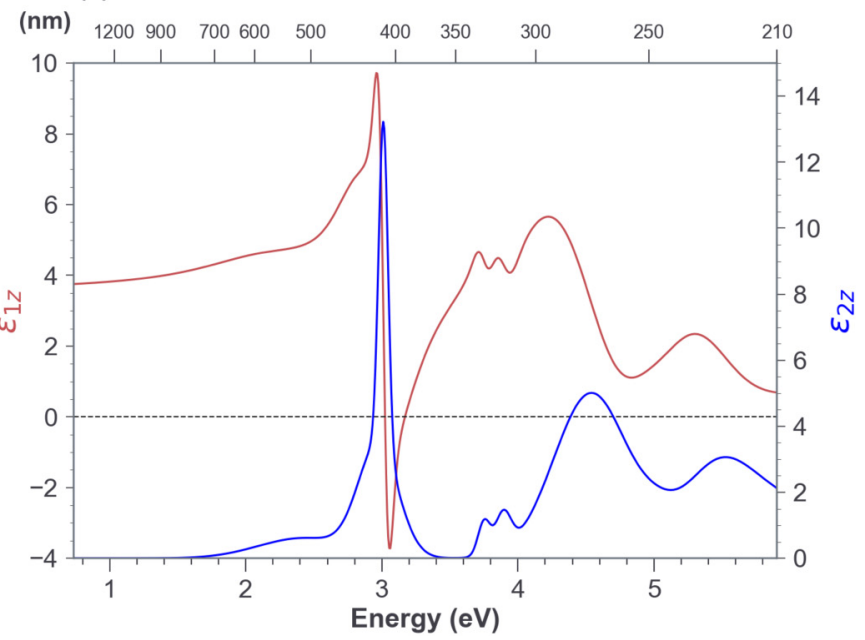

(b): $\varepsilon_{z}$ - SubPc IL

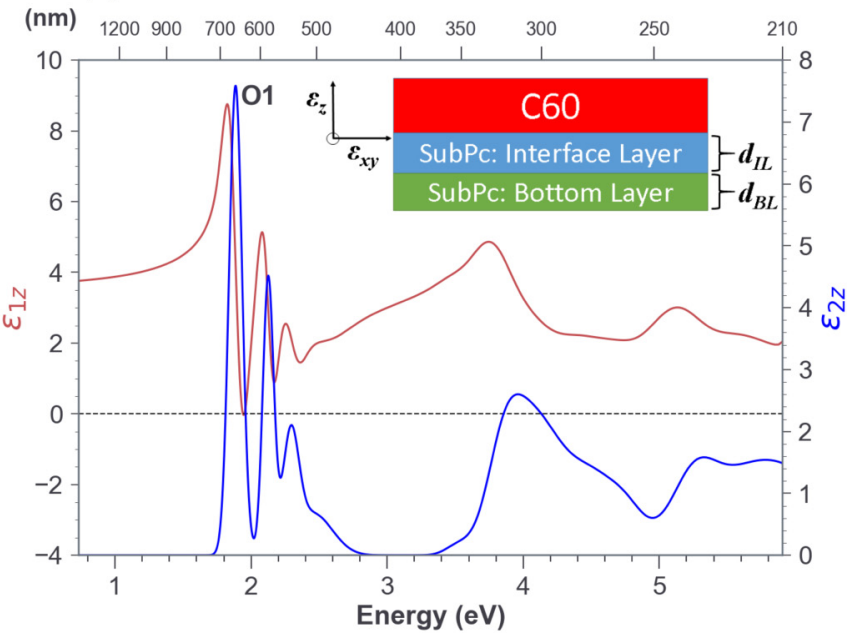

FIG. 5. The out-of-plane dielectric constants, $\epsilon_{z}$, of (a) $12 \mathrm{~nm} \mathrm{C60} \mathrm{layer} \mathrm{on} \mathrm{top} \mathrm{of} \mathrm{SubPc} \mathrm{and} \mathrm{(b)} \mathrm{directly} \mathrm{underlying} \mathrm{(buried)} 1 \mathrm{~nm}$ SubPc interface layer $\left(\mathrm{IL}_{\mathrm{SubPc}}\right.$ ) showing the $\mathrm{O} 1$ transition at $\sim 2 \mathrm{eV}$. The layers correspond to that of Fig. 4 . The inset in (b) shows the schematic of the method used to derive the out-of-plane dielectric constants $\left(\epsilon_{z}\right)$ of the interface layer of SubPc from standard spectroscopic ellipsometry data analysis method (SSE). The buried layer (SubPc here as an example) below C60 is divided into an interface layer with C60 of thickness $d_{I L}$ and a bottom layer $d_{B L} . \epsilon_{x y}$ is the in-plane dielectric constant of a layer.

\section{C60 on phthalocyanines}

Intermolecular interactions in the form of electronic coupling between $\mathrm{C} 60$ and phthalocyanines were next explored starting with SubPc as the bottom layer. C60 was evaporated onto the completed SubPc film (16 nm) of Fig. 4(a) and iSE measurements were carried out during the deposition. The DART analysis is shown in Fig. 4(b). Comparing to the growth of $\mathrm{C} 60$ on an insulating $\mathrm{SiO}_{2}$ substrate (Fig. 1), the data shows strong, anomalous responses centered at 3.6 and $4.8 \mathrm{eV}$ (with a shoulder at $4.6 \mathrm{eV}$ ). Moreover, with increasing deposition of $\mathrm{C} 60$, the response differs from that of $\mathrm{C} 60$ on $\mathrm{SiO}_{2}$. The strength of the $3.6 \mathrm{eV}$ transition increases with deposition of C60 until $6 \mathrm{~nm}$, and remains strong, redshifting and nonzero until the final thickness of $13 \mathrm{~nm}$, while that of the $4.8 \mathrm{eV}$ decreases as in the case of $\mathrm{C} 60$ on $\mathrm{SiO}_{2}$. Since $\delta \rho$ represents the optical response of the layer $\delta d$ that is deposited on the substrate with thin film at thickness $d$, this implies that at C60 thickness $d=0 \mathrm{~nm}$ on top of SubPc, the first incoming C60 molecule interacts (or electronically couples) strongly with SubPc at the $3.6 \mathrm{eV}$ energy corresponding to the primary allowed electronic transition in C60. And as more C60 deposits, a strong overlap and delocalization of the excited state wave functions between the incoming C60 and C60 deposited on SubPc is occurring for the frontier orbital corresponding to the $3.6 \mathrm{eV}$ transition. The redshift of $3.6 \mathrm{eV}$ is more prominent compared to $\mathrm{C} 60$ on $\mathrm{SiO}_{2}$. DART analysis of the same data but calculated for a film thickness change of $\delta d=1 \AA$ is shown in Fig. S4 highlighting the angstrom-level sensitivity of the method.

Additionally, during deposition of C60 on SubPc, a $|\delta \rho|$ response can also be seen as a feature near 2.04-2.05 eV shown in the magnified view in Fig. 4(b).2, which is not present in the $\mathrm{C} 60$ growth on $\mathrm{SiO}_{2}$ in Fig. 1 (and Fig. S2). Thus, it appears that the origin of this feature is in the under- lying SubPc layer. Comparison with the $2.1 \mathrm{eV}$ transition in Fig. 4(a). 2 shows that this feature is redshifted from $2.1 \mathrm{eV}$ by about $\sim 60 \mathrm{meV}$, and appears to correspond to the $\mathrm{O} 1$ transition in Fig. S3(f). The presence of this feature was also investigated by carrying out the DART analysis, calculated for $\delta t=1 \mathrm{~min}$, of the iSE data measured during the downtime between the end of SubPc deposition and start of the C60 deposition. The $\delta \rho$ values of the analysis are shown in Fig. S5, and are essentially zero indicating that there was no other deposition on the SubPc film during this downtime. The weak, noisy features concentrated around 3.6 and $5 \mathrm{eV}$ similar to the energies corresponding to the strong features of SubPc in Fig. 4(a).1 suggests a possible mild rearrangement of SubPc molecules (probably on the surface). This observation also suggests that the DART analysis can be used to directly characterize the stability of a film.

To gain an in-depth understanding of the significance of these anomalous optical responses, systematic analysis of the iSE data was carried out for obtaining the $\epsilon_{z}$ dielectric constants of C60 and the buried SubPc layers using the SSE method. For this, the buried SubPc layer was divided into an interface SubPc layer (IL $\mathrm{ILbPc}_{\mathrm{Su}}$ ) and a bottom SubPc layer $\left(\mathrm{BL}_{\mathrm{SubPc}}\right)$ as shown in the inset in Fig. 5(b) (and Fig. S6). Subsequently, the iSE data at $12 \mathrm{~nm}$ C60 thickness was fit for the out-of-plane dielectric constants $\epsilon_{z}\left(\sqrt{\epsilon_{1 z}+j \epsilon_{2 z}}=\right.$ $n_{z}+j k_{z}$ of refractive index $\mathrm{N}_{z}$ ) of the $12 \mathrm{~nm} \mathrm{C60} \mathrm{and} \mathrm{the}$ $\mathrm{IL}_{\text {SubPc }}$ layer at thickness $d_{\mathrm{ILSubPc}}=1 \mathrm{~nm}$ shown in Fig. 5 (a) and (b) respectively; $d_{\mathrm{BLSubPc}}$ was fixed at $15 \mathrm{~nm}$ with its $\epsilon_{z}$ set to the values before C60 deposition [Figs. S3(e) and $S 3(f)] . \epsilon_{x y}$ of the layers are fixed since the in-plane environment can be reasonably approximated as unvarying with thickness, i.e., $\delta \epsilon_{x y} / \delta d=0$ (see Sec. S2 for more details on the strategy). In contrast to the values of $\mathrm{C} 60$ on $\mathrm{SiO}_{2}$ (Fig. 1) and $\mathrm{SubPc}$ on $\mathrm{SiO}_{2}$ (Fig. S3), the $\epsilon_{2 z}$ of both $\mathrm{C} 60$ and $\mathrm{IL}_{\mathrm{SubPc}}$ here show significant increase at similar energies found in the 
DART analysis, i.e., around 3 and $2 \mathrm{eV}$, respectively. More importantly, the corresponding real part $\epsilon_{1 z}$ becomes negative at these values. This holds significant implications. In metal thin films such as $\mathrm{Au}, \mathrm{Ag}$, and $\mathrm{Al}$, and in conductive oxides such as indium tin oxide, the wavelengths where free electrons absorb are characterized by negative values of $\epsilon_{1}$ with high $\epsilon_{2}$ values due to absorption [14,43,44]. The corresponding frequency of light where $\epsilon_{1 z}$ equals to zero before taking on negative values is called plasma frequency. Hence, observation of such negative values of $\epsilon_{1 z}$ and a concominant rise in $\epsilon_{2 z}$ in both $\mathrm{C} 60$ and SubPc indicates that strong electronic coupling is occurring between the frontier orbitals corresponding to these specific energies, $\sim 3 \mathrm{eV}$ in $\mathrm{C} 60$ and $\sim 2 \mathrm{eV}$ in SubPc, and is leading to resonance electronic transitions in the form of plasmons (or unbound quasiparticles) oscillating between the molecules, and thus the delocalization of the excited state wave functions across the interface. A point to note is that the frontier orbitals corresponding to these excitations are at different energy levels relative to the vacuum level $[40,45,46]$. The $\mathrm{IL}_{\mathrm{SubPc}}$ thickness at resonance will be referred to as $d_{\mathrm{ILSubPc}}^{\text {Res }}$ from here on.

The parameters of the Gaussian oscillators (GO, given by amplitude: Amp, center energy: En, broadening: $\mathrm{Br}$ ) representing the two resonance transitions that were fit to the iSE data had good fit statistics, i.e., C60 GO - Amp: $8.396 \pm$ 0.693, Br: 0.076 \pm 0.006 , En: $2.997 \pm 0.003$; IL $\mathrm{SubPc}_{\mathrm{SO}} \mathrm{GO}-$ Amp: $7.588 \pm 1.361$, Br: $0.112 \pm 0.032$, En: $1.886 \pm 0.024$, with a low mean squared error (MSE 4.0) showing high confidence in the derived $\epsilon_{z}$ values. The value of $d_{\mathrm{ILSubPc}}^{\text {Res }}$ $(1 \mathrm{~nm})$ obtained from SSE analysis, i.e., the depth in the SubPc film until which the resonating wave functions delocalize, is lower than the $4 \mathrm{~nm}$ delocalization distance in SubPc obtained from the DART analysis in Fig. 4(a).2. This is probably because of limitations of the SSE method requiring model fitting of a buried layer, and also lack of additional optical information to decouple the optical properties of the top C60 and the buried SubPc layers further highlighting the significance of the DART analysis method. However, $d_{\mathrm{ILSubPc}}^{\mathrm{Res}}$ can be taken as a lower estimate. Variation of $\epsilon_{z}$ of $\mathrm{IL}_{\mathrm{SubPc}}$ layer for different $d_{\mathrm{ILSubPc}}$ values $(1-16 \mathrm{~nm})$ is shown in Fig. S7, and similarly, variation of $\epsilon_{z}$ of the top C60 layer for its different thicknesses $(9-13 \mathrm{~nm})$ is shown in Fig. S8, Table S1 for fit statistics of the parameters and Sec. $\mathbf{S} 2$ for more details.

In SubPc, this resonance absorption corresponds not to an increase in the $\pi-\pi^{*}$ transition but an increase in its adjacent $\mathrm{O} 1$ transition, which was originally observed in the $\epsilon_{2 z}$ of the bulk film before C60 deposition [see Fig. S3(f)]. The O1 transition of SubPc at which the strong, plasmonic type coupling occurs with $\mathrm{C} 60$ corresponds to excitations into excitonic states. Its energy is below the $\pi-\pi^{*}$ transition in SubPc and in accordance with the energies of the SubPc excitons observed from fluorescence emission measurements $[41,47,48]$. The observed $60 \mathrm{meV}$ Stokes shift in our case, which is about $17 \mathrm{~nm}$ shift at $2.1 \mathrm{eV}$, is in agreement with the $8-30 \mathrm{~nm}$ (depending on the environment) observed in boron-subpthalocyanines [22,41,49-52]. Since the excitons arise from the $\pi-\pi *$ transitions-also reflected in the decrease in $\epsilon_{2 z}$ of the $\pi-\pi^{*}$ transitions at $2.1 \mathrm{eV}$ and a concomitant increase in the $\mathrm{O} 1$ transition at resonance thickness $d_{\mathrm{ILSubPc}}^{\text {Res }}$ in Fig. S7-the electronic coupling, and its evolution, of C60 with the electronic wave function corresponding to the exciton suggests reorganization of SubPc molecules at the interface with C60. These observations are further corroborated by the DART results of C60 deposited on $\mathrm{MoOx} / \mathrm{SubPc}\left(\mathrm{SubPc}_{\mathrm{MoOx}}\right)$ and $\mathrm{MoOx} /$ hexapropyltruxene $(\mathrm{PrT}) / \mathrm{SubPc}\left(\mathrm{SubPc}_{\mathrm{PrT}}\right)$ shown in Figs. S9 and S10 (SSE analysis for $\epsilon_{z}$ are shown in Figs. S7 and S8). Figures S9(b) and $\mathrm{S} 10$ (b) show the $\mathrm{O} 1$ transition at $2.04 \mathrm{eV}$ and electronic coupling of $\mathrm{C} 60$ with SubPc similar to Fig. 4 further highlighting the robustness and strength of the DART analysis method. A major difference in the optical response of SubPc deposited on MoOx and PrT with that on $\mathrm{SiO}_{2}$ can also be noticed at energies above $3 \mathrm{eV}$. We conjecture that this could be due to a possible electronic coupling between SubPc and the underlying electronic materials: $\mathrm{MoOx}$ and PrT, respectively.

In our previous work [22] we showed the use of PrT as an interface layer between $\mathrm{MoOx}$ and SubPc helping reduce exciton quenching leading to an increase in the short-circuit current density $\left(J_{S C}\right)$. Such interlayers are regularly used to improve the performance of organic solar cells $[53,54]$. SSE analysis in that work also showed the $\mathrm{O} 1$ transition in $\epsilon_{2 z}$ of both $\mathrm{SubPc}_{\mathrm{MoOx}}$ and $\mathrm{SubPc}_{\mathrm{PrT}}$ with that of the latter having the highest strength directly corroborating the increased number of excitons in SubPc due to PrT. This increase is also reflected in the increase in $|\delta \rho|$ value at $3.6 \mathrm{eV}$ upon deposition of C60 on SubPc $c_{\text {PrT }}$ [Figs. S9(b).1 and S10(b).1]. With a higher number of excitons available in $S u b P_{P r T}$, higher number of resonance oscillations of the corresponding excited state wave functions are occurring with C60 leading to an increased $|\delta \rho|$ value. This correlation of an increased number of excitons leading to an increase in the resonance $|\delta \rho|$ value is, to an extent, analogous to the observed increase in the absorption at the $\pi-\pi^{*}$ interchain transition from regiorandom P3HT to regioregular P3HT $[55,56]$. The redshifts of the $|\delta \rho|$ peak energies of $\mathrm{C} 60$ as a function of C60 thickness are shown in Fig. S11. The rates of shift are about the same while the peak values for $\mathrm{C} 60 / \mathrm{SubPc}_{\mathrm{PrT}}$, $\mathrm{C} 60 / \mathrm{SubPc}_{\mathrm{MoOx}}, \mathrm{C} 60 / \mathrm{SubPc}_{\mathrm{SiO} 2}$ (of Fig. 4) differ by about $0.1 \mathrm{eV}$. However, compared to that of $\mathrm{C} 60$ on $\mathrm{SiO}_{2}(\sim 18$ $\mathrm{meV} / \mathrm{nm}$ ), the rate of shift is about a factor of 2 higher: $\sim 40 \mathrm{meV} / \mathrm{nm}$.

Finally, to ascertain that this type of electronic coupling phenomenon was not specific to SubPc and C60, experiments and data analyses were carried out for SubNc/C60 bilayer. Results similar to SubPc/C60 were observed, and shown in

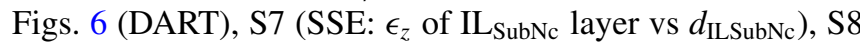
(SSE: $\epsilon_{z}$ vs C60 thickness), S11 (peak shifts), and Table S1 (fit statistics of the O1 transition). Figure 6(a) shows, as for SubPc, firstly, strong electronic coupling, and delocalization, occurring between frontier orbitals of SubNc (deposited on silicon wafer with $4 \mathrm{~nm} \mathrm{MoOx}$ ) at energies above $3 \mathrm{eV}$. For the wave function corresponding to the $\pi-\pi^{*}$ transition, the delocalization distance is about $2-3 \mathrm{~nm}$ after which $|\delta \rho|$ remains approximately constant. Figure 6(b) shows the same type of coupling as for $\mathrm{SubPc} / \mathrm{C} 60$ between the wave function corresponding to SubNc exciton (O1) and that of the $3.6 \mathrm{eV}$ primary transition in $\mathrm{C} 60$, along with delocalization, further corroborating the results.

The plasmonic type absorption $\left(\epsilon_{2 z}\right)$ at very specific energies occurring upon deposition of $\mathrm{C} 60$ on the phthalocyanines 

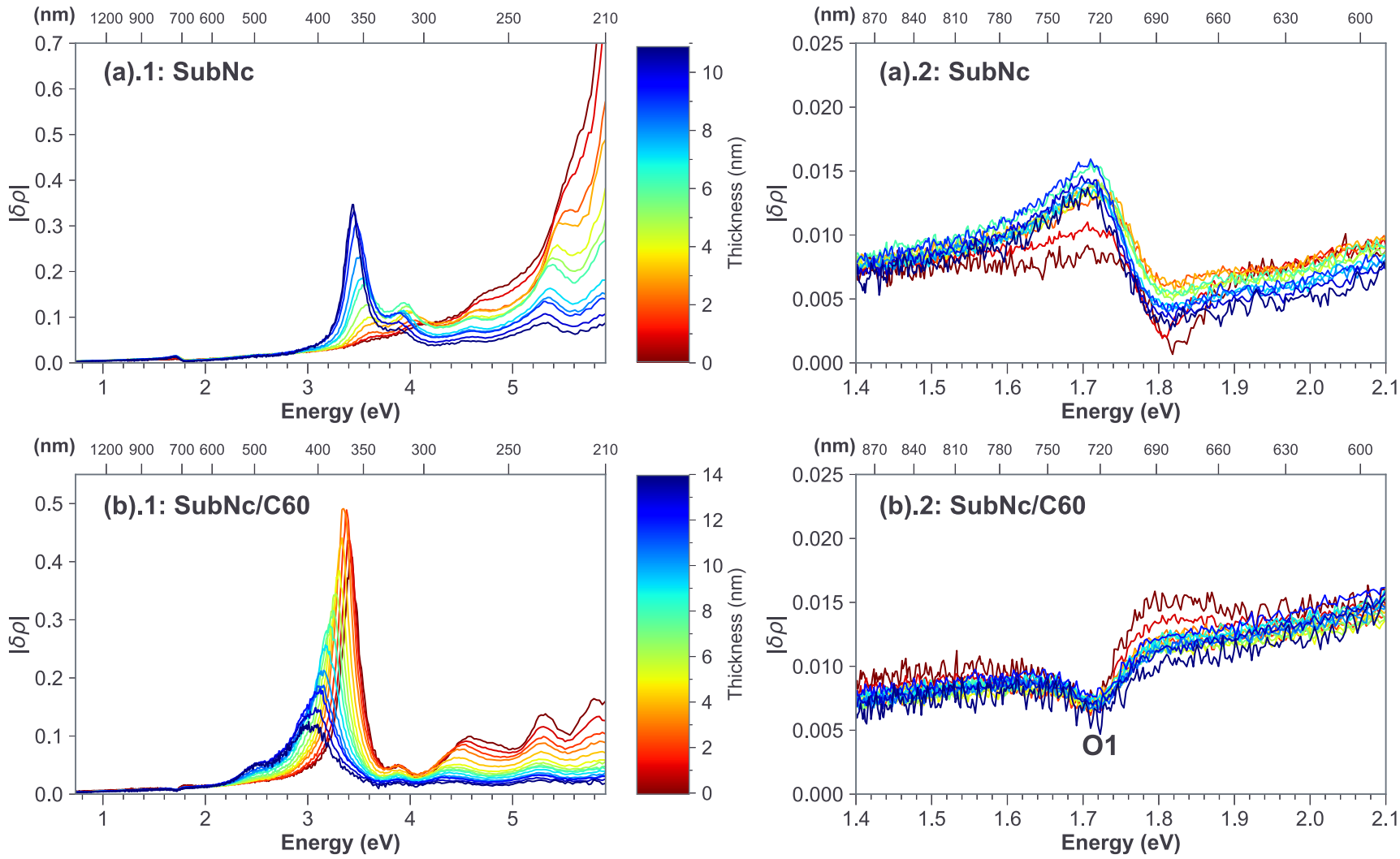

FIG. 6. (a).1 DART analysis of a SubNc film deposited on $\mathrm{Si} / \mathrm{SiO}_{2} / \mathrm{MoOx}$ substrate. (a).2 Magnified view of the same DART results showing the optical response corresponding to the $\pi-\pi^{*}$ transition in SubNc for clear visualization. (b). 1 DART analysis of C60 deposited on the SubNc film in (a). (b).2 Magnified view of the same DART results in (b).1 around $1.8 \mathrm{eV}$. The optical response corresponding to the excitations into SubNc excitonic states is indicated by the label O1. All calculations were carried out for a differential change of $\delta d=1 \mathrm{~nm}$.

implies resonance oscillations of the excited state wave functions between the two materials across the interface. The question of specificity in energies of the frontier orbitals at which electronic coupling is occurring can be partly investigated by examining the transitions in pure C60. The lower energy HOMO $\rightarrow$ LUMO transitions are forbidden in C60 since the states are of the same parity. The primary electronic transitions are ${ }^{1} T_{1 u} \leftarrow{ }^{1} A_{g}$ (or HOMO $\rightarrow$ LUMO + 1) and approximately centered around $3.6,4.6$, and $5.5 \mathrm{eV}$ [25,27-29,57-59], and are also the wave functions that spatially delocalize between C60 molecules as seen in Figs. 1, 4, 6, S9, and S10. From our observations, it appears that the physics dictating the transitions within C60, i.e., Fermi's golden rule [36,37,60], also seems to dictate the transitions from other molecules, arising from resonance oscillations, into C60. More specifically, the question of why electronic coupling is occurring at $3.6 \mathrm{eV}$ and not the other primary transitions is difficult to answer at this point. We can only conjecture that the frontier orbitals corresponding to the $3.6 \mathrm{eV}$ primary transition is closest in energy, relative to the vacuum level, to that of the $\pi-\pi^{*}$ transition in the phthalocyanines. This coupling might have consequences for exciton dissociation from phthalocyanine to C60 and vice versa. In light of these intriguing observations, understanding their implications in the perspective of quantum mechanics and solar cell device physics is of pivotal importance, and will be explored in our next work with further donors and acceptors including nonfullerene acceptors, and at different substrate temperatures to explore the effects of molecular orientation on electronic coupling. The method will also be applied to test for Fermi-level pinning arising due to electronic coupling between organic semiconductors and metal electrodes.

\section{CONCLUSIONS}

We have shown a method, called DART, for analyzing in situ spectroscopic ellipsometry data yielding direct information representative of the optical properties (dielectric constants) primarily along the growth direction of vacuumdeposited thin films in real time without the need for model fitting. Application of this analysis method to the study of pristine organic semiconductor thin films, C60 and MeO-TPD on $\mathrm{SiO}_{2}$, showed electronic coupling and delocalization of the excited state wave functions corresponding to the frontier orbitals. Extending the same to the growth of C60 on phthalocyanines (SubPc and SubNc), strong, anomalous optical features were observed in $\mathrm{C} 60$ at energies corresponding to its primary allowed transition at $3.6 \mathrm{eV}$, which increased and then decreased in strength while redshifting with C60 deposition. Accompanying features were observed in the underlying phthalocyanine layers at energies corresponding to their excitons. Dielectric constants along the growth or thickness direction of the bilayer films derived from systematic analysis using standard ellipsometry data analysis method 
(model fitting) revealed such features to be plasmonic type oscillations of the excited state wave functions corresponding to phthalocyanine excitons and the $3.6 \mathrm{eV}$ primary transition of $\mathrm{C} 60$, the strength of which changed with the number of excitons. Spatial delocalization (along the thickness direction) of the wave functions in C60 and phthalocyanines was also observed.

These results using DART have revealed singular insights into the physics of fullerene C60 and phthalocyanines in the thickness direction, and thus understanding the implications on organic optoelectronics device physics such as organic solar cells will be crucial. In the case of deposition techniques where in situ growth measurements are not possible such as solution-processing methods, the DART method can still be used to study post-treatment effects, e.g., DART analysis could give insights into effects of structural changes on the optical properties, for example, from thermal treatment, and consequently structure-property relationships and stability can be examined. And finally, strength of the presented analysis method as displayed in its simplicity, angstrom-level sensitivity, robustness, and reproducibility, could be highly useful in conjunction with other in situ optical spectroscopy methods $[9,12]$ in examining thin film systems comprising of semiconductors, quantum materials or any other materials where interfacial electronic coupling is highly crucial in determining the device properties.
The research materials (iSE data) supporting this publication can be publicly accessed on the Oxford University Research Archive via the following digital object identifier: [61] The research materials are available under a CC BY license.

\section{ACKNOWLEDGMENTS}

S.V.K. would like to thank the AFMD group, headed by M.K.R. at Oxford, especially A. Jungbluth, A. E. Lauritzen, Dr. A. Privitera, I. Habib, Dr. P. Kaienburg, Dr. T. Derrien for the valuable discussions and feedback on the manuscript. S.V.K. and M.K.R. would also like to thank Prof. P. Skabara, School of Chemistry, University of Glasgow for the hexapropyltruxene. S.V.K. and M.K.R. express their gratitude to EPSRC (WAFT, Grants No. EP/M015173/1; IAA and No. EP/R511742/1) and UKRI (START, Grant No. ST/R002754/1) for funding.

S.V.K. developed the DART method, and conceptualized and executed the study. Discussion of the results and planning the experiments were carried out by S.V.K. and M.K.R.. The first draft was written by S.V.K.. All authors participated in the preparation and review.

Oxford University Innovation has filed a UK patent application related to the DART method of this manuscript. Application No.: 2002576.3, Status: Pending.
[1] N. W. Ashcroft and N. D. Mermin, Solid State Physics (Brooks/Cole, Thomson Learning, South Melbourne; London, 1976).

[2] S. H. Simon, The Oxford Solid State Basics (Oxford University Press, Oxford, 2013).

[3] M. Azzouzi, J. Yan, T. Kirchartz, K. Liu, J. Wang, H. Wu, and J. Nelson, Nonradiative Energy Losses in Bulk-Heterojunction Organic Photovoltaics, Phys. Rev. X 8, 031055 (2018).

[4] F. D. Eisner, M. Azzouzi, Z. Fei, X. Hou, T. D. Anthopoulos, T. J. S. Dennis, M. Heeney, and J. Nelson, Hybridization of local exciton and charge-transfer states reduces nonradiative voltage losses in organic solar cells, J. Am. Chem. Soc. 141, 6362 (2019).

[5] S. Athanasopoulos, H. Bässler, and A. Köhler, Disorder vs delocalization: Which is more advantageous for high-efficiency Organic Solar Cells? J. Phys. Chem. Lett. 10, 7107 (2019).

[6] F.-J. Kahle, C. Saller, S. Olthof, C. Li, J. Lebert, S. Weiß, E. M. Herzig, S. Hüttner, K. Meerholz, P. Strohriegl, and A. Köhler, Does electron delocalization influence charge separation at donor-acceptor interfaces in organic photovoltaic cells? J. Phys. Chem. C 122, 21792 (2018).

[7] S. M. Menke, N. A. Ran, G. C. Bazan, and R. H. Friend, Understanding energy loss in organic solar cells: toward a new efficiency regime, Joule 2, 25 (2018).

[8] S. Gélinas, A. Rao, A. Kumar, S. L. Smith, A. W. Chin, J. Clark, T. S. Van Der Poll, G. C. Bazan, and R. H. Friend, Ultrafast long-range charge separation in organic semiconductor photovoltaic diodes, Science 343, 512 (2014).

[9] R. Forker, M. Gruenewald, and T. Fritz, Optical differential reflectance spectroscopy on thin molecular films, Annual Reports Section "C" (Physical Chemistry) 108, 34 (2012).
[10] M. Gruenewald, J. Peuker, M. Meissner, F. Sojka, R. Forker, and T. Fritz, Impact of a molecular wetting layer on the structural and optical properties of tin(II)-phthalocyanine multilayers on Ag(111), Phys. Rev. B 93, 115418 (2016).

[11] D. E. Aspnes and A. A. Studna, Anisotropies In The AboveBand-Gap Optical Spectra Of Cubic Semiconductors, Phys. Rev. Lett. 54, 1956 (1985).

[12] D. S. Martin and P. Weightman, Reflection anisotropy spectroscopy of molecular assembly at metal surfaces, Thin Solid Films 455-456, 752 (2004), the 3rd International Conference on Spectroscopic Ellipsometry.

[13] G. Bussetti, S. Cirilli, A. Violante, V. Chiostri, C. Goletti, P. Chiaradia, A. Sassella, M. Campione, L. Raimondo, D. Braga, and A. Borghesi, Reflectance anisotropy spectroscopy: A probe to explore organic epitaxial growth, Journal of Vacuum Science \& Technology A 27, 1029 (2009).

[14] H. Fujiwara, Spectroscopic Ellipsometry : Principles and Applications (John Wiley, Chichester, 2007).

[15] R. M. A Azzam and N. M. Bashara, Ellipsometry and Polarized Light (Oxford, North-Holland, Amsterdam, NY, 1977).

[16] K. Hinrichs, M. Levichkova, D. Wynands, K. Walzer, K. J. Eichhorn, P. Bäuerle, K. Leo, and M. Riede, Morphology and molecular orientation of ethyl-substituted dicyanovinylsexithiophene films for photovoltaic applications, Thin Solid Films 525, 97 (2012).

[17] D. Yokoyama, Molecular orientation in small-molecule organic light-emitting diodes, J. Mater. Chem. 21, 19187 (2011).

[18] M. F. G. Klein, G. Q. G. de Medeiros, P. Kapetana, U. Lemmer, and A. Colsmann, Modeling approach to derive the anisotropic complex refractive index of polymer:fullerene blends for or- 
ganic solar cells utilizing spectroscopic ellipsometry, J. Photon. Energy 5, 057204 (2015).

[19] C. Schünemann, D. Wynands, K.-J. Eichhorn, M. Stamm, K. Leo, and M. Riede, Evaluation and control of the orientation of small molecules for strongly absorbing organic thin films, J. Phys. Chem. C 117, 11600 (2013).

[20] G. F. Burkhard, E. T. Hoke, and M. D. McGehee, Accounting for interference, scattering, and electrode absorption to make accurate internal quantum efficiency measurements in organic and other thin solar cells, Adv. Mater. 22, 3293 (2010).

[21] D. Yokoyama and C. Adachi, In situ real-time spectroscopic ellipsometry measurement for the investigation of molecular orientation in organic amorphous multilayer structures, J. Appl. Phys. 107, 123512 (2010).

[22] H. Ye, S. V. Kesava, J. F. M. Hardigree, R. E. Brown, G. Mazzotta, R. Warren, P. J. Skabara, and M. Riede, Efficiency enhancement of small molecule organic solar cells using hexapropyltruxene as an interface layer, J. Mater. Chem. C 8, 4909 (2020).

[23] T. Heitz and B. Drévillon, In situ infrared ellipsometry study of the growth of hydrogenated amorphous carbon thin films, Thin Solid Films 313-314, 704 (1998).

[24] See Supplemental Material at http://link.aps.org/supplemental/ 10.1103/PhysRevMaterials.5.015601 for a basic derivation of $\delta \rho$; standard spectroscopic ellipsometry (SSE) analysis description and issues; derived dielectric constants; fit statistics of the O1 transition parameters; DART results of $\mathrm{C} 60$ on $\mathrm{SiO}_{2}$, time gap between SubPc and C60, 660 on $\mathrm{SiO}_{2} / \mathrm{SubPc}$ calculated for $\delta d=1 \AA$, C60 on MoOx/SubPc and PrT/SubPc; and $|\delta \rho|$ peak energy values of C60 vs C60 thickness deposited on the different SubPc films and SubNc film.

[25] S. Leach, M. Vervloet, A. Desprès, E. Bréheret, J. P. Hare, T. John Dennis, H. W. Kroto, R. Taylor, and D. R. M. Walton, Electronic spectra and transitions of the fullerene c60, Chem. Phys. 160, 451 (1992).

[26] A. Sassara, G. Zerza, M. Chergui, and S. Leach, Absorption wavelengths and bandwidths for interstellar searches of c 60 in the 2400-4100 a region, Astrophys. J., Suppl. Ser. 135, 263 (2001).

[27] A. Lucas, G. Gensterblum, J. J. Pireaux, P. A. Thiry, R. Caudano, J. P. Vigneron, P. Lambin, and W. Krätschmer, Elementary excitations of c24 from the far infrared to the far vacuum ultraviolet studied by high-resolution electron-energyloss spectroscopy, Phys. Rev. B 45, 13694 (1992).

[28] V. S. Pavlovich and E. M. Shpilevsky, Absorption and fluorescence spectra of c24 fullerene concentrated solutions in hexane and polystyrene at 77-300 k, J. Appl. Spectrosc. 77, 335 (2010).

[29] R. Fukuda and M. Ehara, Electronic excitations of c24 fullerene calculated using the $a b$ initio cluster expansion method, J. Chem. Phys. 137, 134304 (2012).

[30] A. A. Bakulin, A. Rao, V. G. Pavelyev, P. H. M. van Loosdrecht, M. S. Pshenichnikov, D. Niedzialek, J. Cornil, D. Beljonne, and R. H. Friend, The role of driving energy and delocalized states for charge separation in organic semiconductors, Science $\mathbf{3 3 5}$, 1340 (2012).

[31] Q. Burlingame, C. Coburn, X. Che, A. Panda, Y. Qu, and S. R. Forrest, Centimetre-scale electron diffusion in photoactive organic heterostructures, Nature 554, 77 (2018).
[32] B. Bernardo, D. Cheyns, B. Verreet, R. D. Schaller, B. P. Rand, and N. C. Giebink, Delocalization and dielectric screening of charge transfer states in organic photovoltaic cells, Nat. Commun. 5, 3245 (2014).

[33] H. Kobayashi, S. Hattori, R. Shirasawa, and S. Tomiya, Wannier-like delocalized exciton generation in c24 fullerene clusters: A density functional theory study, J. Phys. Chem. C 124, 2379 (2020).

[34] E. E. Jelley, Spectral absorption and fluorescence of dyes in the molecular state, Nature 138, 1009 (1936).

[35] G. Scheibe, Über die veränderlichkeit der absorptionsspektren in lösungen und die nebenvalenzen als ihre ursache, Angew. Chem. 50, 212 (1937).

[36] A. Köhler and H. Bassler, Electronic Processes in Organic Semiconductors (Wiley-VCH, Weinheim, 2015).

[37] M. Pope and C. E. Swenberg, Electronic Processes of Organic Crystals and Polymers (Clarendon Press, Oxford, 1999).

[38] M. L. Tietze, L. Burtone, M. Riede, B. Lüssem, and K. Leo, Fermi level shift and doping efficiency in p-doped small molecule organic semiconductors: A photoelectron spectroscopy and theoretical study, Phys. Rev. B 86, 035320 (2012).

[39] R. Meerheim, S. Olthof, M. Hermenau, S. Scholz, A. Petrich, N. Tessler, O. Solomeshch, B. Lüssem, M. Riede, and K. Leo, Investigation of c60f36 as low-volatility p-dopant in organic optoelectronic devices, J. Appl. Phys. 109, 103102 (2011).

[40] S. Olthof, R. Timmreck, M. Riede, and K. Leo, Photoelectron spectroscopy investigations of recombination contacts for tandem organic solar cells, Appl. Phys. Lett. 100, 113302 (2012).

[41] G. E. Morse and T. P. Bender, Boron subphthalocyanines as organic electronic materials, ACS Appl. Mater. Interfaces 4, 5055 (2012).

[42] H. M. Rhoda, M. P. Kayser, Y. Wang, A. Y. Nazarenko, R. V. Belosludov, P. Kiprof, D. A. Blank, and V. N. Nemykin, Tuning up an electronic structure of the subphthalocyanine derivatives toward electron-transfer process in noncovalent complexes with c24 and c70 fullerenes: Experimental and theoretical studies, Inorg. Chem. 55, 9549 (2016).

[43] S. Adachi, The Handbook on Optical Constants of Metals (World Scientific, Singapore, 2012).

[44] D. W. Lynch and W. R. Hunter, Comments on the Optical Constants of Metals and an Introduction to the Data for Several Metals, in Handbook of Optical Constants of Solids, edited by Edward D Palik (Academic Press, Burlington, 1997) pp. 275-367.

[45] S. W. Cho, L. F. J. Piper, A. DeMasi, A. R. H. Preston, K. E. Smith, K. V. Chauhan, P. Sullivan, R. A. Hatton, and T. S. Jones, Electronic structure of C60/Phthalocyanine/ITO interfaces studied using soft X-ray spectroscopies, J. Phys. Chem. C 114, 1928 (2010).

[46] K. Cnops, G. Zango, J. Genoe, P. Heremans, M. V. MartinezDiaz, T. Torres, and D. Cheyns, Energy level tuning of nonfullerene acceptors in organic solar cells, J. Am. Chem. Soc. 137, 8991 (2015).

[47] H. Gommans, S. Schols, A. Kadashchuk, P. Heremans, and S. C. J. Meskers, Exciton diffusion length and lifetime in subphthalocyanine films, J. Phys. Chem. C 113, 2974 (2009).

[48] C.-F. Lin, V. M. Nichols, Y.-C. Cheng, C. J. Bardeen, M.K. Wei, S.-W. Liu, C.-C. Lee, W.-C. Su, T.-L. Chiu, H.-C. Han, Li.-C. Chen, C.-Ti. Chen, and J.-H. Lee, Chloroboron subphthalocyanine/C60 planar heterojunction organic solar cell 
with N,N-dicarbazolyl-3,5-benzene blocking layer, Sol. Energy Mater. Sol. Cells 122, 264 (2014).

[49] R. A. Kipp, J. A. Simon, M. Beggs, H. E. Ensley, and R. H. Schmehl, Photophysical and photochemical investigation of a dodecafluorosubphthalocyanine derivative, J. Phys. Chem. A 102, 5659 (1998).

[50] G. E. Morse, M. G. Helander, J. F. Maka, Z.-H. Lu, and T. P. Bender, Fluorinated phenoxy boron subphthalocyanines in organic light-emitting diodes, ACS Appl. Mater. Interfaces 2, 1934 (2010).

[51] G. E. Morse, J. S. Castrucci, M. G. Helander, Z.-H. Lu, and T. P. Bender, Phthalimido-boronsubphthalocyanines: New derivatives of boronsubphthalocyanine with bipolar electrochemistry and functionality in OLEDs. ACS Appl. Mater. Interfaces 3, 3538 (2011).

[52] M. G. Helander, G. E. Morse, J. Qiu, J. S. Castrucci, T. P. Bender, and Z.-H. Lu, Pentafluorophenoxy boron subphthalocyanine as a fluorescent dopant emitter in organic light emitting diodes, ACS Appl. Mater. Interfaces 2, 3147 (2010).

[53] Z. Yin, J. Wei, and Q. Zheng, Interfacial materials for organic solar cells: Recent advances and perspectives, Adv. Sci. 3, 1500362 (2016)

[54] K. J. Bergemann, J. A. Amonoo, B. Song, P. F. Green, and S. R. Forrest, Surprisingly high conductivity and efficient exciton blocking in fullerene/wide-energy-gap small molecule mixtures, Nano Lett. 15, 3994 (2015).

[55] P. J. Brown, D. S. Thomas, A. Köhler, J. S. Wilson, J.-S. Kim, C. M. Ramsdale, H. Sirringhaus, and R. H. Friend, Effect of interchain interactions on the absorption and emission of poly(3-hexylthiophene), Phys. Rev. B 67, 064203 (2003).

[56] F. C. Spano and C. Silva, H- and J-aggregate behavior in polymeric semiconductors, Annu. Rev. Phys. Chem. 65, 477 (2014).

[57] G. Orlandi and F. Negri, Electronic states and transitions in C60 and C70 fullerenes, Photochemical \& Photobiological Sciences 1, 289 (2002).

[58] A. L. Montero-Alejo, E. Menendez-Proupin, M. E. Fuentes, A. Delgado, F.-P. Montforts, L. A. Montero-Cabrera, and J. M. Garcia de la Vega, Electronic excitations of C60 aggregates, Phys. Chem. Chem. Phys. 14, 13058 (2012).

[59] R. Bauernschmitt, R. Ahlrichs, F. H. Hennrich, and M. M. Kappes, Experiment versus time dependent density functional theory prediction of fullerene electronic absorption, J. Am. Chem. Soc. 120, 5052 (1998).

[60] P. A. M. Dirac, The quantum theory of the emission and absorption of radiation, Proc. R. Soc. Lond. A 114, 243 (1927).

[61] https://doi.org/10.5287/bodleian:KzjB5BnPb. 